\title{
Research Paper: Methadone Maintenance Treatment in Iran and Brief Psychological Treatments for Women: A Rehabilitation Approach in Methamphetamine Dependence
}

\author{
Armin Esmaili ${ }^{1}\left(\mathbb{C}\right.$, Sara Shishehgar $^{2} \mathbb{1},{ }^{*}$ Omid Massah $^{3}$ (1)
}

1. Shokufa Drug Abuse Treatment Center, Tehran, Iran.

2. Women's and New Born Health Department, Westmead Hospital, Sydney, Australia.

3. Substance Abuse and Dependence Research Center, University of Social Welfare and Rehabilitation Sciences, Tehran, Iran.

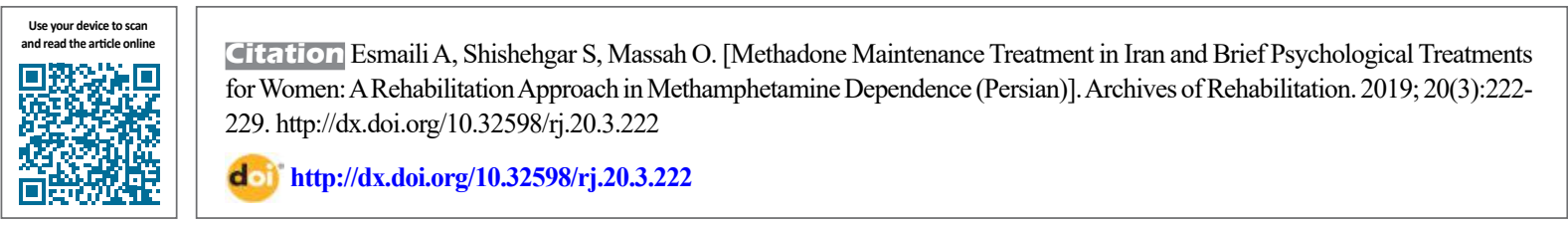

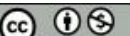

Received: 27 May 2019 Accepted: 25 Aug 2019 Available Online: 01 Oct 2019

Keywords: Addiction, Rehabilitation, Psychological treatment, Methamphetamine, Women, Short-term intervention, Methadone maintenance treatment

\section{ABSTRACT}

Objective Methamphetamine use in patients treated with methadone is a health problem in Iran that reduces the benefits of this treatment. This has been more reported by women than by men. Short-term psychological interventions are one of the major methods of rehabilitation to solve this problem. The current study aimed to explore the reasons for methadone patients for using these interventions to quit daily MA use while in treatment.

Materials \& Methods In this qualitative-descriptive study, 64 women undergoing maintenance treatment from three methadone clinics in Tehran, Iran, were selected through a simple and accessible method and were interviewed.in 2016. All women met the Diagnostic and Statistical Manual, Edition Five (DSM-V) criteria for regular MA use in the past 12 months while in methadone treatment. A researcher-made questionnaire was devised to interview with the patients. Quantitative-descriptive data were analyzed by PPSS software V. 22 and qualitative data were analyzed by content analysis method.

Results The mean age of the women was 37.8 years (age range: $18-56$ years). Overall, $50 \%$ of the participants reported living with their families. The average duration of undergoing methadone treatment was 18 months. Overall, $84.3 \%$ of participants consumed MA by smoking. The main reasons women expressed as self-reporting to use short-term psychological interventions were as follows: learning short-sighted cognitive skills is easy and affordable to quit smoking (79\%): routine counseling and training on methadone treatment does not have a significant effect on stopping the use of MA, as it requires cognitive-behavioral skills and techniques (76\%); with continued consumption, there is a possibility of increased divorce and separation from the family and consequently, lack of financial support (71\%), which caused lack of paying for methadone treatment charges; therefore, learning cognitive-behavioral skills was necessary; regular MA use is against the religious and traditional values of family and society (66\%); likelihood of homelessness was high due to continued MA use (53\%); the stigma and social labeling and discrimination against consumption for women are higher than men (51\%); positive feedback of methadone-therapy has been greatly reduced due to consuming MA and therefore the probability of expulsion from methadone-therapy is high $\mathrm{P}(43 \%)$. Problems related to work $(38 \%)$ and risk of losing the right to foster care (31\%) were also other reasons.

Conclusion A combination of individual, familial, social and therapeutic reasons were described by MA dependent women as their needs for having short-term psychological treatments while experiencing methadone treatment. More studies should be conducted to investigate the effects of short-term psychological treatments in reducing regular MA use among women in methadone treatment services. Family therapy needs to be provided to reduce the likelihood of divorce, separation, homelessness, and lack of financial support among these women. Mass media should provide educational programs to reduced stigma and discrimination against women with regular MA use.

\section{* Corresponding Author:}

Omid Massah, MD.

Address: Substance Abuse and Dependence Research Center, University of Social Welfare and Rehabilitation Sciences, Tehran, Iran.

Tel: +98 (21) 71732830

E-Mail: omchomch@gmail.com 


\title{
درمان نتَهدارنده با متادون در ايران و درمانهاى روانشناختى كوتاهمدت براي زنان: يك رويكرد بازتوانى در درمان وابستكى بله شيشه
}

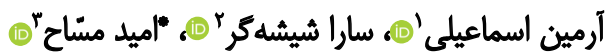

1- ا- مركز درمان سوهمصرف مواد شكوفا، تهران، ايران.

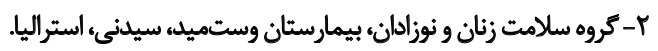

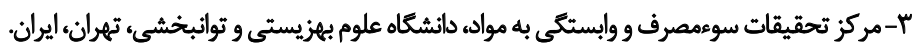

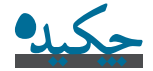

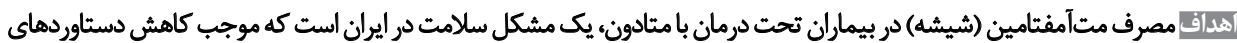

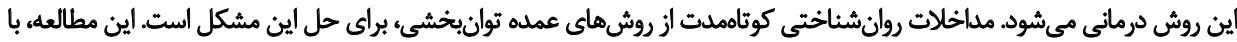

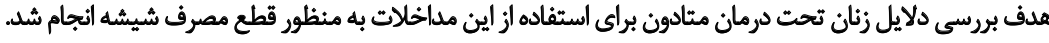

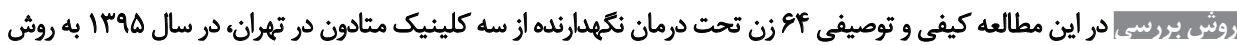

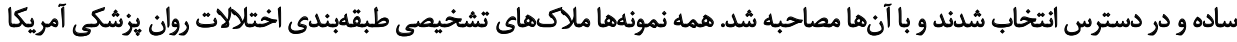

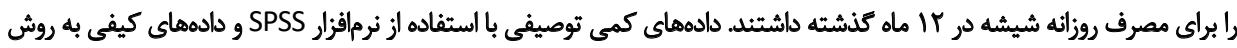

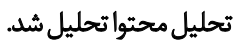

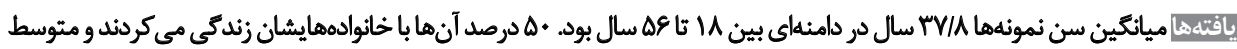

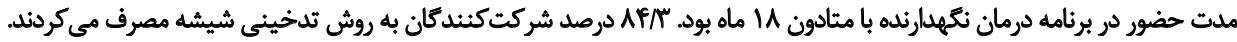

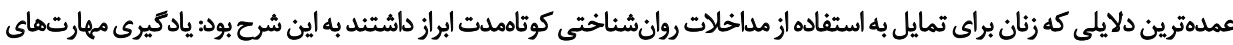

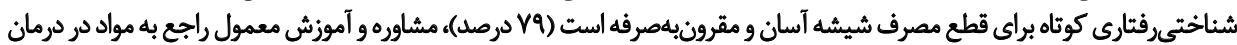

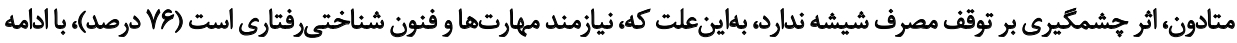

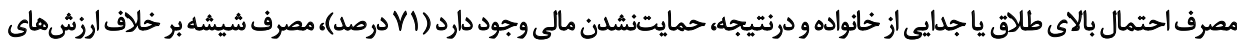

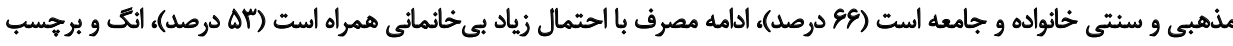

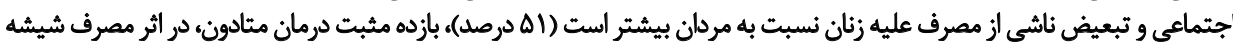

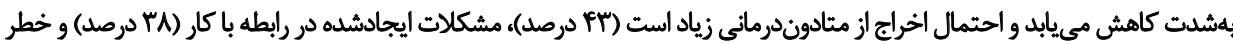

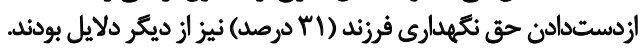

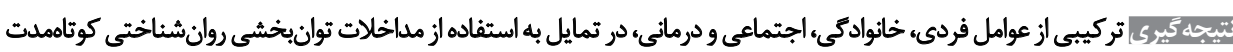

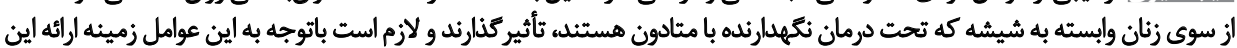

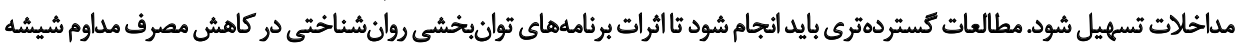

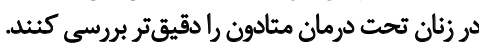

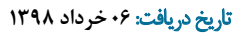

تاريخ يذيرش: ب. شهريور

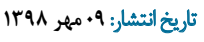

مصرف شيشه در زنان تحت درمان نكُهدارنده با متادون بسيار

dales

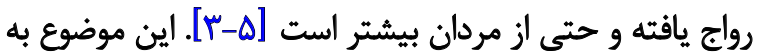

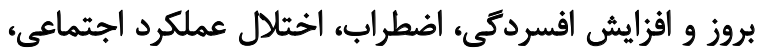

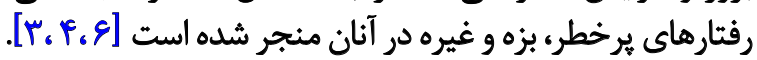

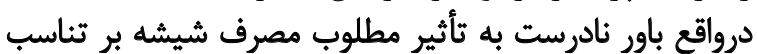

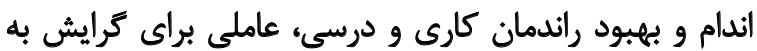
مصرف ماده محرك متآمفتامين' (شيشه)، بازده مثبت

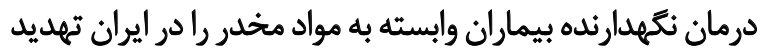

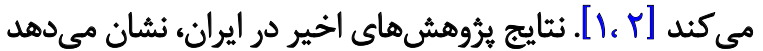

1. Methamphetamine

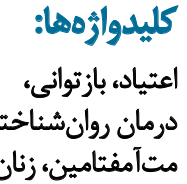

$$
\begin{aligned}
& \text { * نويسينده مسيئول: } \\
& \text { دكتر اميد مناح } \\
& \text { نشائي: تهران، دانشكاه علوم بهزيستي و توانبخشي، مركز تحقيقات سوموصرف و وابستكي به مواد. }
\end{aligned}
$$

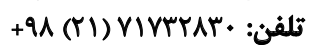

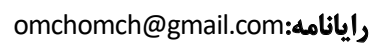


ملاكهاى تشخيصى طبقهبندى اختّلالات روانيزشكى آمريكاه

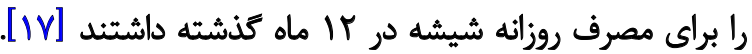

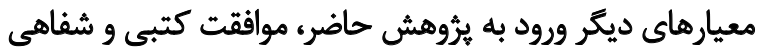

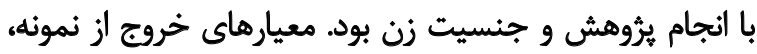

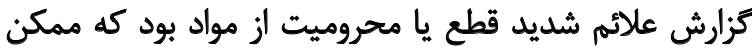

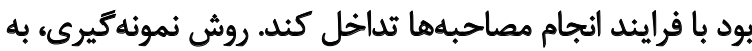

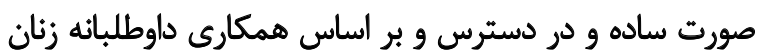

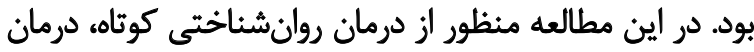

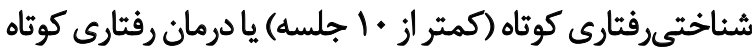
(كمتر از • ا جلسه) بوده است.

مصاحبهشوندكان از محرمانهبودن ئروهش و حفظ إن اصل

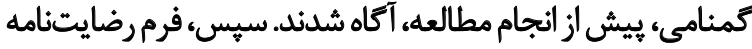

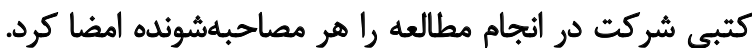

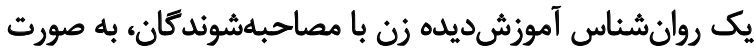

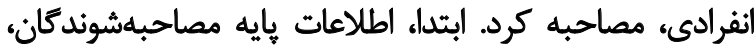

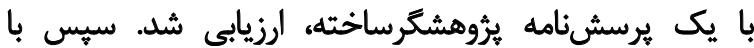

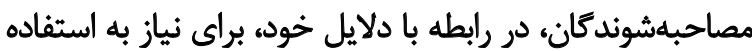

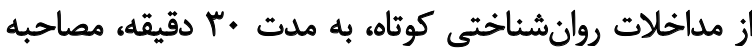

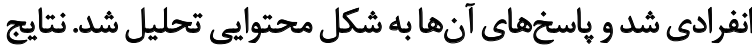

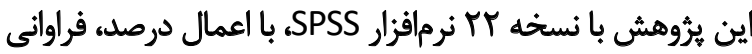
و ميانكين، تجزيه و تحليل آمارى شد.

ياقتهنها

اطلاعات بايه مصاحبهشوندكان، در جدول شماره ا، تزارش

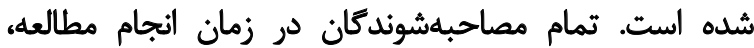

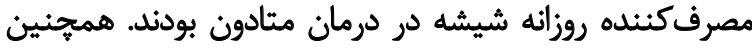

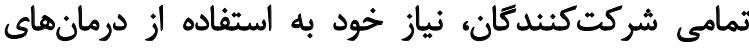

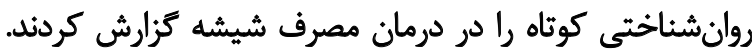

مهمترين دلايل بيانشده عبارت بودند ازئ دران

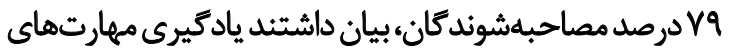

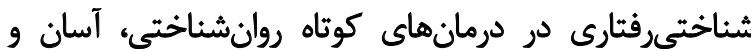
مقرونبهصرفهاست.

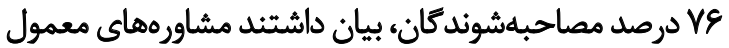

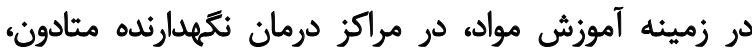

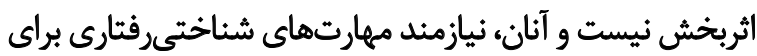
كنترل وسوسه و جلوكيرى از لغزش هستند.

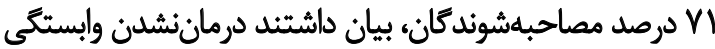

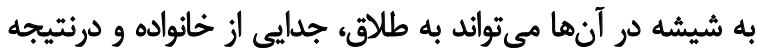

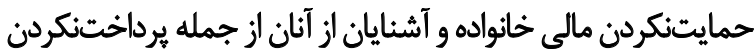

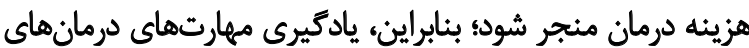
شناختىرفتارى كوتاه براى آنها لازم است.

5.Diagnostic and Statistical Manual of Mental Disorders (DSM-V)

$$
\text { مصرف شيشه از سوى زنان است [V]]. }
$$

متأسفانه درمان دارويى خاصى براى مصرف شيشه وجود ندارد

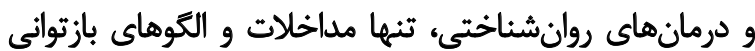

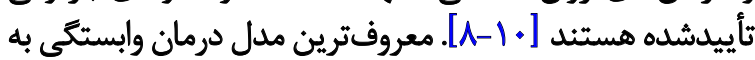

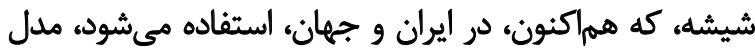

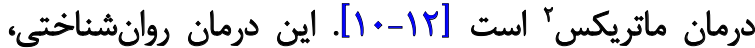

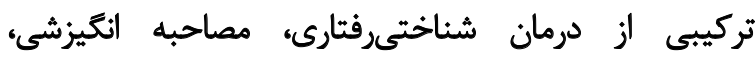

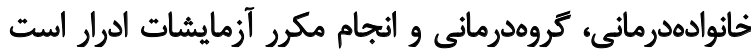

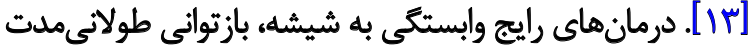

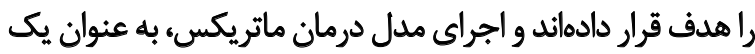

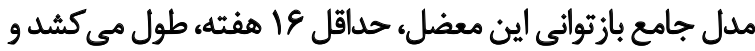

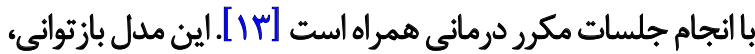

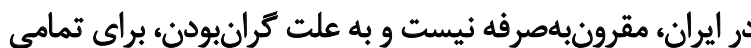

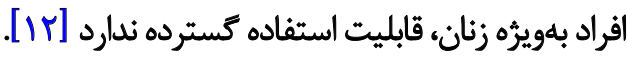

اثربخشى مدل هاى مختلف درمانهاى شناختىرفتارى، در درمان

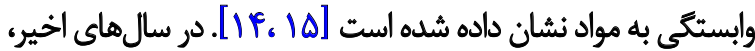

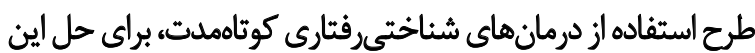

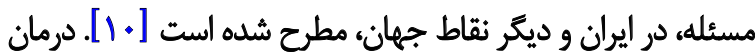

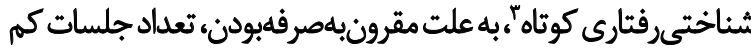

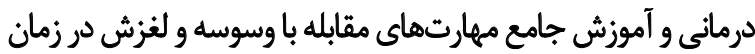

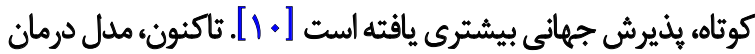

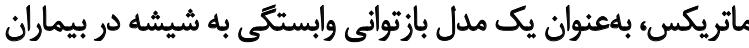

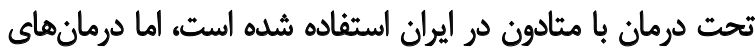

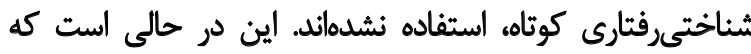

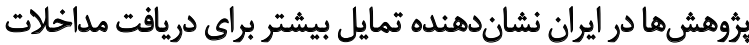

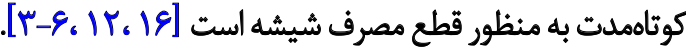

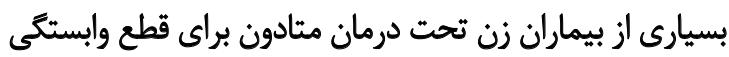

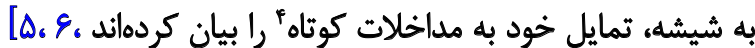

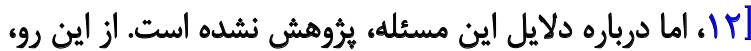

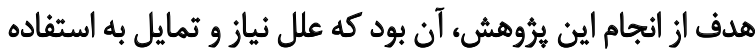

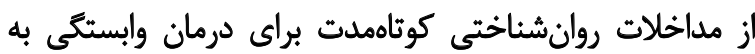

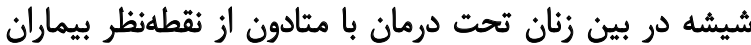
بررسى شود. و طرح يرؤش حاضر كيفى و توصيفى است و به روش تحليل

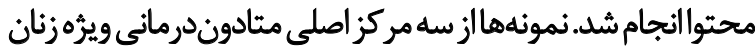

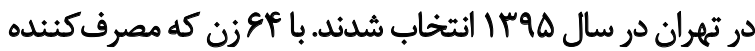
روزانه شيشه در درمان متادون بودند، مصاحبه شد شد تمامى زنان،

\section{Matrix mode}

3. Brief cognitive-behavioural treatment

4. Brief 
جدول (.اطلاعات يايه مصاحبهشوندكان (تعداد نمونه: f4 ئفر)

\begin{tabular}{|c|c|c|}
\hline آماره & & \\
\hline $\operatorname{se}(1+\infty)$ & & جنسيث (زن) (درصل) \\
\hline$r / A$ & & ميانكين سنى (سال) \\
\hline$\Delta$ & & محلدوده سنى (سال) \\
\hline Irts & & محدوده تحصيلات (سال) \\
\hline $\begin{array}{l}M(F / / F) \\
M(\Delta) / F)\end{array}$ & اززواجكرده & وضعيت أزدواج (در حال حاضر) (درصد) \\
\hline $\begin{array}{l}M\left(\Delta_{*}\right) \\
M\left(\Delta_{*}\right)\end{array}$ & بل با خانواده & وضعيت زندكى (در حال حاضر) (درصد) \\
\hline$M$ & & متوسط مدتزمان بودن در مثادوندمانى (ماه) \\
\hline$\Delta$ & & هتوسط مدتزمان وابستكى به شيشه (سال) \\
\hline $\begin{array}{l}\Delta f(A \& / Y) \\
1 .(1 \Delta / M)\end{array}$ & تلدخينى توراكى & روش مصرف شيشه در مثادوندرمانى (درصد) \\
\hline
\end{tabular}

توانبخنتى

و هرصد مصاحبهشوندكان، بيان داشتئل مصرف شيشه بر

درمان رايج وابستكّي به شيشه يك بازتواني طولاتيمدت و

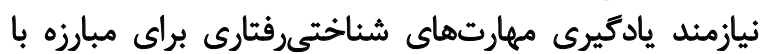

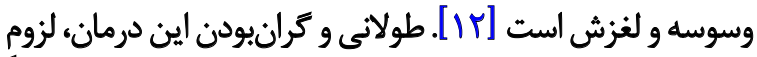

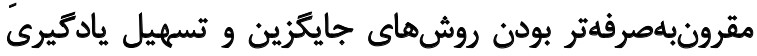

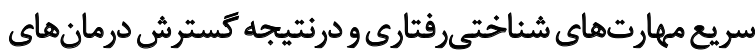

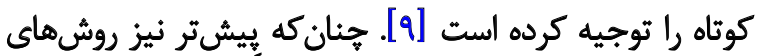

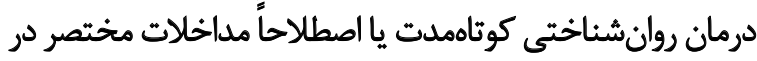

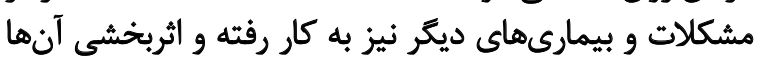

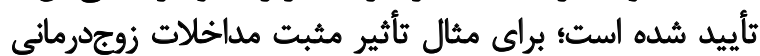

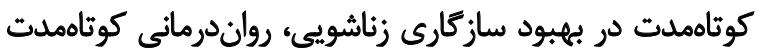

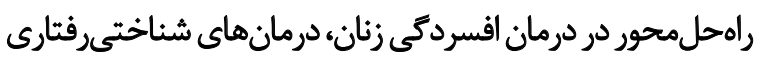

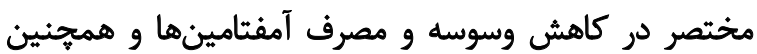

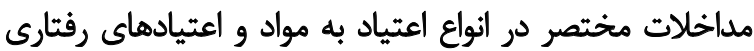

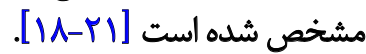

مطالعات نشان ميدهد زنان بهتر از مردان به درمان

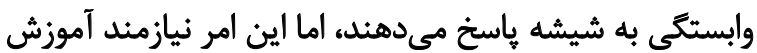

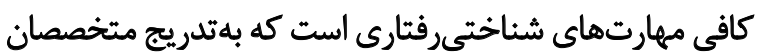

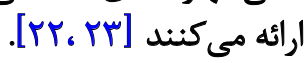

شركت كنئدكان در اين مطالعه، مجموعهاي از عوامل فردى،

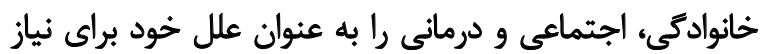

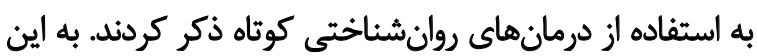

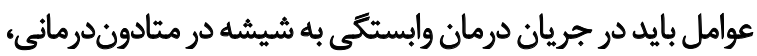

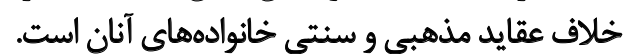
هr درصد مصاحبهشوندكان، بيان داشتند مصرف شيشه

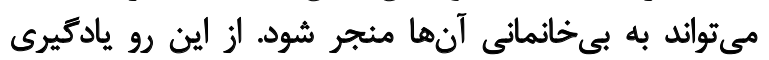

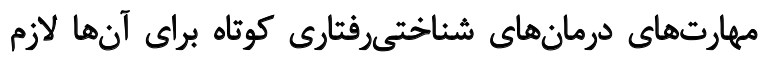
أست.

|ه درصد مصاحبهشوندكان، بيان داشتيند برجسب اجتماعي و

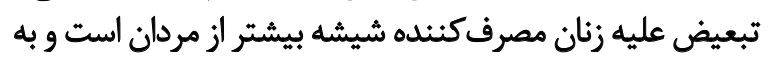
همين دليل، آنها نيازمئد اين درمان هان هستيند.

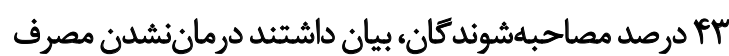

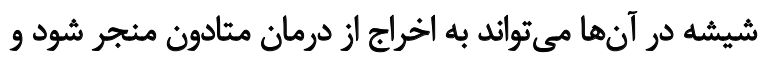

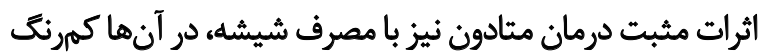

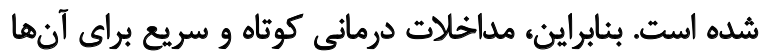
لازم است. - ماست.

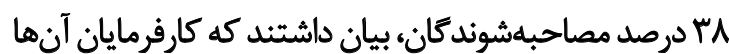

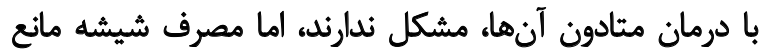

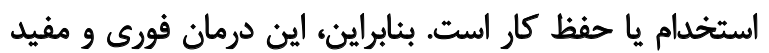
براي آنها لازم است.

اب درصد مصاحبهشوندكان، بيان داشتند كه مصرف شيشه

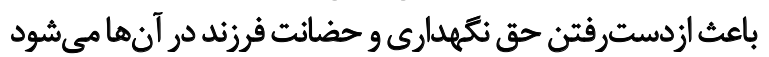

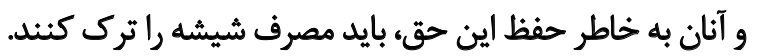


مطالعه بود. از اين رو يرُوهشهاى بزركت بتر با تعداد نمونه بيشتر

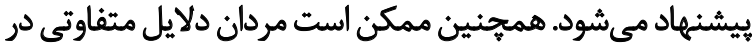

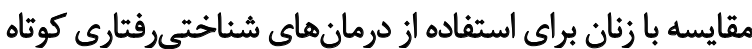

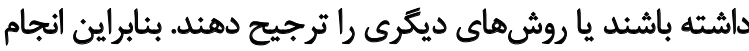

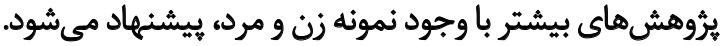

\section{مالاحظات اخلاقي}

\section{ييروى از اصول اخلاق يُوهشي}

اين مطالعه راكميته اخلاق دانشعاه علوم بهزيستى وتوانبخشى إنى

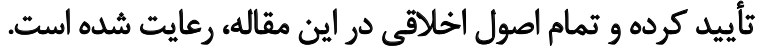

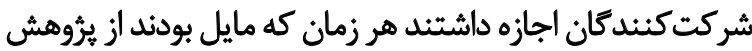

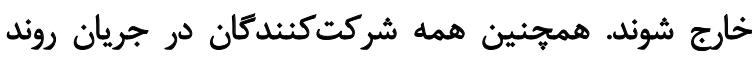
يُروهش بودند و اطلاعات آنها، محرمانه نكَّه داشته شد شدي.

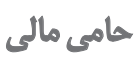

اين مقاله حمايت مالى نداشته است.

$$
\text { مشاركت نويسند مكَان }
$$

مفهومسازى، طراحى و روششناسى: سارا شيشهكر و اميد

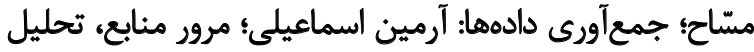

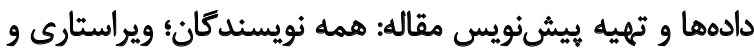
نهاييىسازى نوشته: اميد متّاح

$$
\text { تعارض مثافع }
$$

بنا بر اظهار نويسندكان، اين مقاله، تعارض منافع ندارد.
توجه شود. توجهنكردن به اين دلايل مى توانئد به عنوان عوامل

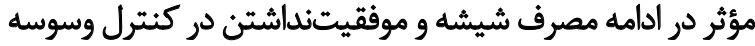

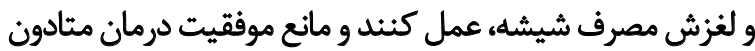

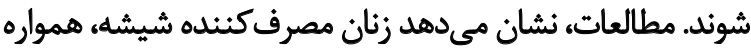

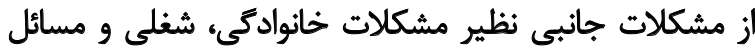

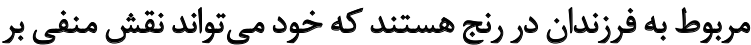

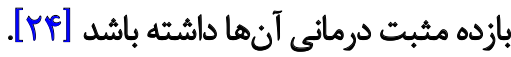

شيوع بالاى مصرف شيشه در بيماران تحت درمان با متادون و

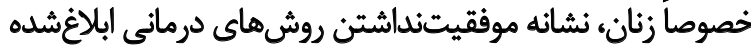

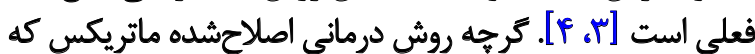

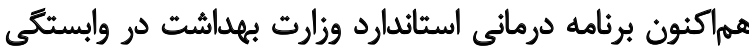

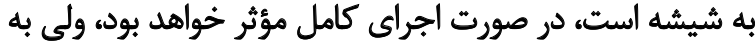

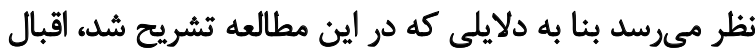

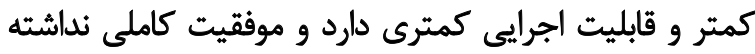

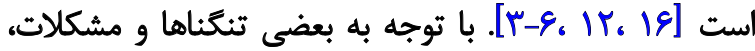

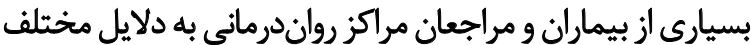

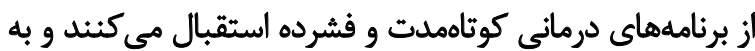

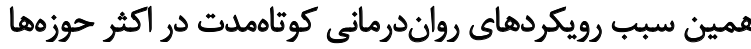

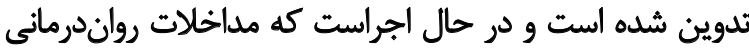

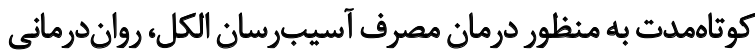

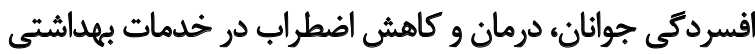

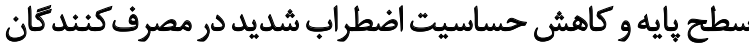

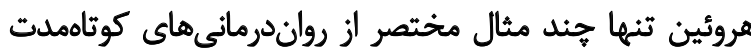

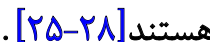

تثيجلئيرى

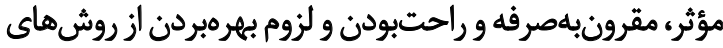
درمانى كوتامدت

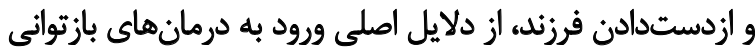

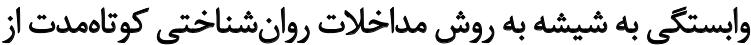

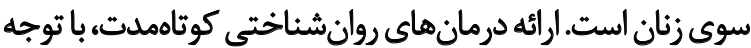

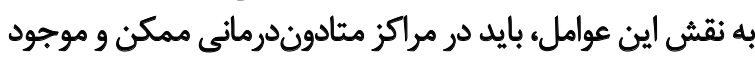

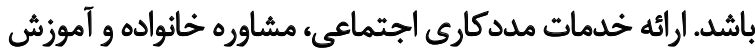

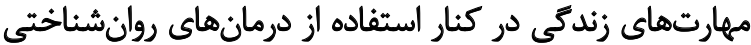

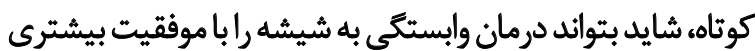

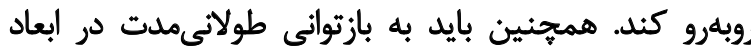

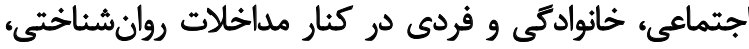
توجه تخصصى وحرفهاي شود. مطالعه حاضر اولين مطالعه در ايران، در رابطه با دلايل

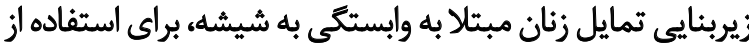

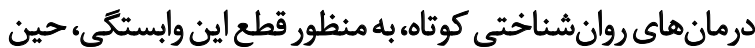

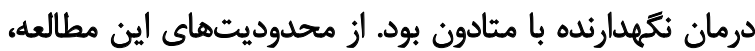
تعداد كم نمونه، تعداد كم مراكز نمونهيَيرى و ماهيت توصيفى إنى 


\section{References}

[1] Radfar SR, Cousins SJ, Shariatirad S, Noroozi A, Rawson RA. Methamphetamine use among patients undergoing methadone maintenance treatment in Iran: A threat for harm reduction and treatment strategies: A qualitative study. International Journal of High Risk Behaviors and Addiction. 2016; 5(4):0-0.

[2] Shariatirad S, Maarefvand M, Ekhtiari H. Methamphetamine use and methadone maintenance treatment: An emerging problem in the drug addiction treatment network in Iran. International Journal of Drug Policy. 2013; 24(6):e115-e6. [DOI:10.1016/j.drugpo.2013.05.003] [PMID]

[3] Massah O, Moradi A. The Prevalence of methamphetamine dependence among Iranian women in methadone maintenance therapy in Tehran, Iran. Iranian Journal of Psychiatry. 2018; 13(1):10-4.

[4] Massah O, Shishehgar S. Methamphetamine dependence, psychological well-being, criminality and high risk sexual behaviors in female-only methadone services in Tehran and Karaj, Iran. Iranian Journal of Psychiatry and Behavioral Sciences. 2018; 12(2):e61859. [DOI:10.5812/ijpbs.61859]

[5] Dana S, Effatpanah M, Mahjoub A. The new epidemic problem of psychoactive drugs at drug treatment centers of Iran: Implications for education, prevention and treatment. Iranian Journal of Psychiatry and Behavioral Sciences. 2018; 12(2):e63555. [DOI:10.5812/ijpbs.63555]

[6] Roshanfekr P, Noori R, Dejman M, Fathi Geshnigani Z, Rafiey H. Drug use and sex work among at-risk women: A qualitative study of initial factors. Iranian Journal of Psychiatry and Behavioral Sciences. 2015; 9(2):e953-e. [DOI:10.17795/ ijpbs953] [PMID] [PMCID]

[7] Massah O, Farhoudian A, Noori R, Ghaderi S, Ahounbar E, Mousavi SH. Relationship between social, economic and cultural factors and women's tendency toward crystal methamphetamine or opium use. Iranian Rehabilitation Journal. 2018; 16(3):247-54. [DOI:10.32598/irj.16.3.247]

[8] Lee NK, Jenner L, Harney A, Cameron J. Pharmacotherapy for amphetamine dependence: A systematic review. Drug and Alcohol Dependence. 2018; 191:309-37. [DOI:10.1016/j. drugalcdep.2018.06.038] [PMID]

[9] Radfar SR, Rawson RA. Current research on methamphetamine: Epidemiology, medical and psychiatric effects, treatment, and harm reduction efforts. Addiction \& Health. 2014; 6(3-4):146-54. [PMID] [PMCID]

[10] Mehrjerdi ZA. A brief overview of methamphetamine use treatment in Iran: Intervention and practice. Journal of Research in Medical Sciences. 2013; 18(12):1018-20. [PMID] [PMCID]

[11] Massah O, Effatpanah M, Shishehgar S. Matrix model for methamphetamine dependence among Iranian female methadone patients: The first report from the most populated Persian Gulf country. Iranian Rehabilitation Journal. 2017; 15(3):193-8. [DOI:10.29252/nrip.irj.15.3.193]

[12] Sami S, Effatpanah M, Moradi A, Massah O. Matrix model as an intensive rehabilitation in three methadone services in Iran. Iranian Rehabilitation Journal. 2017; 15(3):293-8. [DOI:10.29252/nrip.irj.15.3.293]

[13] Taymoori P, Pashaei T. Relapse and risk-taking among Iranian methamphetamine abusers undergoing matrix treatment model. Addiction \& Health. 2016; 8(1):49-60.

[14] Doustian Y, Shafi'e-Abadi A, Kalantar-Koushe SM, Massah O. Effectiveness of Marlatt's cognitive-behavioral model on increasing self-efficacy in opiate-dependents. Archives of Rehabilitation. 2014; 14(0):38-48.

[15] Karami H, Khakbaz H, Sohrabi F, Barati-Sedeh F, Farhadi MH. [Effectiveness of cognitive-behavioral group therapy for anger management in reducing wife abuse and increasing marital satisfaction in patients with substance abuse (Persian)]. Archives of Rehabilitation. 2014; 14(S1):91-9.

[16] Rafiey H, Ghaderi S, Morovat B, Noori R, Effatpanah M, Mahjoub A, et al. Amphetamine type stimulants use in the adult population of Tehran: implications for long term rehabilitation. Iranian Rehabilitation Journal. 2017; 15(4):303-8. [DOI:10.29252/nrip.irj.15.4.303]

[17] Association AP. Diagnostic and Statistical Manual of Mental Disorders (DSM- $\left.{ }^{\circledR}\right)$. Washington, D.C.: American Psychiatric Publishing; 2013.

[18] Davoodi Z, Etemadi O, Bahrami F, Shahsiah M. The effect of brief solution-focused couple therapy approach on couples' marital adjustment in men and women prone to divorce in 2010-2011 in Isfahan. Journal of Fundamentals of Mental Health. 2012; 14(55):190-9.

[19] Dashtizadeh N, Sajedi H, Nazari A, Davarniya R, Shakarami M. Effectiveness of Solution-Focused Brief Therapy (SFBT) on reducing symptoms of depression in women. Journal of Clinical Nursing and Midwifery. 2015; 4(3):67-78.

[20] Srisurapanont M, Sombatmai S, Boripuntakul T. Brief intervention for students with methamphetamine use disorders: A randomized controlled trial. American Journal on Addic tions. 2007; 16(2):111-6. [DOI:10.1080/10550490601184431] [PMID]

[21] DiClemente CC, Corno CM, Graydon MM, Wiprovnick AE, Knoblach DJ. Motivational interviewing, enhancement, and brief interventions over the last decade: A review of reviews of efficacy and effectiveness. Psychology of Addictive Behaviors. 2017; 31(8):862-87. [DOI:10.1037/adb0000318] [PMID]

[22] Hser Yl, Evans E, Huang YC. Treatment outcomes among women and men methamphetamine abusers in California. Journal of Substance Abuse Treatment. 2005; 28(1):77-85. [DOI:10.1016/j.jsat.2004.10.009] [PMID]

[23] Harada T, Tsutomi H, Mori R, Wilson DB. Cognitive-behavioural treatment for Amphetamine-Type Stimulants (ATS)-use disorders. Cochrane Database of Systematic Reviews. 2018 2018(12):CD011315. [DOI:10.1002/14651858.CD011315] [PMID] [PMCID]

[24] Greenfield SF, Back SE, Lawson K, Brady KT. Substance abuse in women. The Psychiatric Clinics of North America. 2010; 33(2):339-55. [DOI:10.1016/j.psc.2010.01.004] [PMID] [PMCID] 
[25] Johnson M, Jackson R, Guillaume L, Meier P, Goyder E. Barriers and facilitators to implementing screening and brief intervention for alcohol misuse: A systematic review of qualitative evidence. Journal of Public Health. 2010; 33(3):41221. [DOI:10.1093/pubmed/fdq095] [PMID]

[26] Wood A, Harrington R, Moore A. Controlled trial of a brief cognitive-behavioural intervention in adolescent patients with depressive disorders. Journal of Child Psychology and Psychiatry. 1996; 37(6):737-46. [DOI:10.1111/j.1469-7610.1996. tb01466.x] [PMID]

[27] Roy-Byrne P, Veitengruber JP, Bystritsky A, Edlund MJ, Sullivan $G$, Craske MG, et al. Brief intervention for anxiety in primary care patients. The Journal of the American Board of Family Medicine. 2009; 22(2):175-86. [DOI:10.3122/jab$\mathrm{fm} .2009 .02 .080078]$ [PMID] [PMCID]

[28] Tull MT, Schulzinger D, Schmidt NB, Zvolensky MJ, Lejuez CW. Development and initial examination of a brief intervention for heightened anxiety sensitivity among heroin users. Behavior Modification. 2007; 31(2):220-42. [DOI:10.1177/0145445506297020] [PMID] 
This Page Intentionally Left Blank 